\title{
FISIOTERAPIA RESPIRATÓRIA NA PNEUMATOCELE: DISCUTINDO EVIDÊNCIAS
}

\section{RESPIRATORY PHYSIOTHERAPY IN PNEUMATOCELE: DISCUSSING EVIDENCE}

Paloma L. F. Parazzi (ORCID: 0000-0002-7072-603X) Juliana Cardoso (ORCID: 0000-0002-2371-8416)

Fabiula J. Mata Belém ( ORCID: 0000-0002-0688-8747)²

Janaina C. Scalco (ORCID: 0000-0002-5230-8213) ${ }^{2}$

Andrezza B. D áquino (ORCID: 0000-0002-6577-846) ${ }^{3}$

Camila Isabel Santos Schivinski (ORCID: 0000-0002-6139-9727)2

\section{RESUMO}

Introdução: a fisioterapia respiratória tem sido elencada no manejo de pacientes com pneumatocele com o objetivo de manter a integridade pulmonar e prevenir complicações causadas pela ruptura da parede da cavidade e da instalação de processo infeccioso nela. No entanto, são poucas as evidências dessa terapêutica, sendo relevante a apresentação da literatura existente sobre o tema. Objetivo: verificar as pesquisas que contemplaram o uso de fisioterapia respiratória em pacientes com diagnóstico de pneumatocele e sua repercussão terapêutica. Métodos: a revisão de literatura foi realizada por meio de pesquisa nas bases de dados Literatura Latino-Americana e do Caribe em Ciências da Saúde (Lilacs), PubMed (Ovid) e Physiotherapy Evidence Database (PEDro), acessadas até abril de 2019. Utilizaram-se combinações entre palavras-chave: pneumatocele, lung abscess, pneumonia, lung cystic, bullous lung disease. Resultados: identificou-se um total de 988 artigos, dos quais foram elencados 93 títulos relacionados com o assunto; destes, foram selecionados 16 estudos para avaliação dos resumos, cuja leitura direcionou para eleição de 6 artigos na íntegra para compor a presente revisão. Conclusões: os artigos selecionados evidenciam efeitos positivos da aplicação de recursos e técnicas fisioterapêuticas em indivíduos com pneumatocele, como melhora da função pulmonar, diminuição de sintomas e melhora da qualidade de vida. Porém, ainda se faz necessária a realização de estudos controlados, com amostras satisfatórias, para comprovação da segurança e eficácia desse procedimento terapêutico.

Palavras-chave: Pneumatocele; Abscesso pulmonar; Pneumonia; Pulmão cístico; Doença pulmonar bolhosa.

Autor correspondente:

Camila Isabel Santos Schivinski

E-mail: cacaiss@yahoo.com.br
${ }^{1}$ Faculdade de Ciências Médicas, Universidade estadual de Campinas

${ }^{2}$ Programa de Pós-graduação em Fisioterapia, Universidade do Estado de Santa Catarina.

${ }^{3}$ Curso de Fisioterapia, Universidade do Estado de Santa Catarina.

\begin{abstract}
Introduction: respiratory physiotherapy has been listed in the management of patients with pneumatocele in order to maintain pulmonary integrity and to prevent complications caused by rupture of the cavity wall and the installation of an infectious process in it. However, there is little evidence of this therapy, being relevant the presentation of existing literature on the subject. Objective: to verify the studies that contemplated the use of respiratory physiotherapy in patients with pneumatocele diagnosis and its therapeutic repercussion. Methods: the literature review was carried out by means of a search in the databases Latin American and Caribbean Literature in Health Sciences (Lilacs), PubMed (Ovid) and Physiotherapy Evidence Database (PEDro), accessed through April 2019. Keyword combinations were used: pneumatocele, lung abscess, pneumonia, lung cystic, bullous lung disease. Results: a total of 988 articles were identified, of which 93 titles related to the subject were listed, and from these, 16 studies were selected for the evaluation of abstracts, whose reading led to the selection of six articles in the whole to compose the present review. Conclusion: the selected articles show positive effects of the application of physiotherapeutic resources and techniques in individuals with pneumatocele, such as improvement of lung function, reduction of symptoms and improvement of quality of life. However, it is still necessary to carry out controlled studies, with satisfactory samples, to prove the safety and efficacy of this therapeutic procedure.
\end{abstract}

Keywords: Pneumatocele; Lung abscess; Pneumonia; Lung cystic; Bullous lung disease. 


\section{INTRODUÇ̃̃O}

A pneumatocele é caracterizada pela presença de cistos cheios de ar nos pulmões, que podem ocorrer quando uma lesão ou inflamação brônquica criam uma válvula de retenção na entrada do ar no parênquima pulmonar ${ }^{1,2}$, formando, assim, pequenos abscessos. Tais cistos/abscessos são geralmente de paredes finas e resultam na destruição tecidual ${ }^{3}$.

A união desses pequenos cistos/ abscessos, também chamados de áreas de necrose do tecido intersticial bronquiolar, resulta na penetração da parede brônquica. Após a formação dessas cavidades, ocorre o desenvolvimento acelerado do mecanismo de insuflação das válvulas .

Existem diversas causas para o desenvolvimento das pneumatoceles, sendo que, entre as mais comuns na infância, está a pneumonia causada pela bactéria Staphylococos 5 . Relata-se que, nessa faixa etária, de acordo com Hoorebeke et al. ${ }^{6}$, as crianças são aparentemente mais vulneráveis às pneumatoceles traumáticas, pois a elasticidade da caixa torácica, que permite a transmissão das forças externas ao parênquima pulmonar, favorece o aprisionamento de ar em uma área de laceração pulmonar após trauma local.

Segundo Fujii e Moulton ${ }^{7}$, em lactentes prematuros, o enfisema pulmonar intersticial pode evoluir para pneumatocele, porém, em neonatos, a formação de pneumatocele é uma complicação muito rara8. Outros fatores relatados para as causas da pneumatocele são: aspiração de hidrocarbonetos, tuberculose e complicação da ventilação com pressão positiva que aparecem mais frequentemente em pacientes com Doença Pulmonar Obstrutiva Crônica (DPOC) $)^{9,10}$.

A presença das pneumatoceles pode ser identificada tanto por exames físicos quanto por exames de imagem ${ }^{3}$. No exame físico, observa-se que os sintomas iniciais, muitas vezes sutis e inespecíficos como dor no peito, tosse e dispneia, podem ser observados dentro dos primeiros dias ${ }^{11}$. Já nos exames de imagem, voltados para diagnósticos, a radiografia de tórax seria um recurso capaz de verificar se há a presença de espaço aéreo cístico ${ }^{12}$.

A tomografia computadorizada de tórax permite uma avaliação mais detalhada para identificação de complicações e realização de diagnósticos diferenciais e mais precisos, tanto para pneumotórax como presença de cistos pulmonares ${ }^{13,14,16}$. Além desses recursos, a ressonância magnética também pode ser utilizada para apontar outras complicações como a hemorragia pulmonar ${ }^{17,18}$.

De acordo com a literatura, a maioria das pneumatoceles tem resolução completa e sem sequelas dentro de semanas a meses, no entanto, algumas persistem e necessitam de monitorização intermitente até a sua resolução ${ }^{18}$. A maioria dos estudos sugere que o tratamento seja conservador, com acompanhamento rigoroso; e técnicas minimamente invasivas devem ser reservadas para pacientes que desenvolvem complicações ${ }^{19}$.

No entanto, o papel da fisioterapia respiratória no tratamento das pneumatoceles ainda não está claro, mas acredita-se que algumas técnicas possam ser aplicadas para diminuir os sintomas respiratórios e melhorar a qualidade de vida desses indivíduos. Diante do exposto, o presente artigo tem como objetivo apresentar uma revisão sistemática de literatura referente ao tratamento fisioterapêutico respiratório utilizado para o manejo de indivíduos com pneumatocele. 
Para a estruturação desta revisão, foram realizadas buscas sistematizadas em três bases de dados eletrônicas: 1) Lilacs - Literatura Latino-Americana e do Caribe em Ciências da Saúde; 2) PubMed (Ovid); e 3) PEDro - Physiotherapy Evidence Database, todas acessadas até abril de 2019.
Estratégias originais de busca foram elaboradas pelos autores para a base de dados Lilacs e PubMed, apresentadas na figura 1 , devido à ausência de publicação sobre esse tema. Para a base de dados PEDro, foram utilizadas as palavras-chave: pneumatocele, abcesso pulmonar, lung abscess.

Figura 1. Quadro elaborado pelos autores apresentando as estratégias de busca nas bases de dados PubMed (via Ovid), Lilacs e PEDro

\begin{tabular}{|c|c|}
\hline \multicolumn{2}{|l|}{ PubMed via Ovid } \\
\hline 1. "Pneumatocele" & 14. "Physiotherapy" \\
\hline 2. "Lung Abscess" & 15. "Therapeutic Exercise" \\
\hline 3. "Lung cysts" & 16. "Exercise" \\
\hline 4. "Bullous lung disease" & 17. "Child" \\
\hline 5. "Empyema" & 18. "Pediatric" \\
\hline 6. "Empyema pulmonary" & 19. "Adolescents" \\
\hline 7. "Pneumonia, Bacterial" & 20. "School age" \\
\hline 8. "Pneumonia staphyococcal" & 21. "Infant" \\
\hline 9. "Pneumonia complicated" & 22. 1 or 2 or 3 or 4 or 5 or 6 or 7 or 8 or 9 \\
\hline 10. "Respiratory Therapy" & 23. 10 or 11 or 12 or 13 or 14 or 15 or 16 \\
\hline 11. "Physical Therapy" & 24. 17 or 18 or 19 or 20 or 21 \\
\hline 12. "Chest Physical Therapy" & 25. 22 and 23 and 24 \\
\hline \multicolumn{2}{|l|}{ 13. "Combined Modality Therapy " } \\
\hline \multicolumn{2}{|l|}{ LILACS } \\
\hline \multicolumn{2}{|c|}{$\begin{array}{l}\text { (tw:(pneumocistic)) OR (tw:(pneumonia por pneumocistic)) OR (tw:(pneumonia)) } \\
\text { OR (tw:(pneumonia, bacterial)) OR (tw:(pneumonia stafilocócica)) OR (tw:(abscesso } \\
\text { pulmonar)) OR (tw:(empyema )) OR (tw:(pneumatocele)) OR (tw:(doença pulmonar } \\
\text { bolhosa)) AND (tw:(respiratory therapy)) OR (tw:(physical therapy)) OR (tw:(physical } \\
\text { therapy modalities)) OR (tw:(physical therapy speciality)) OR (tw:(physiotherapy } \\
\text { (techniques))) OR (tw:(exercise therapy)) OR (tw:(breathing exercise)) }\end{array}$} \\
\hline \multicolumn{2}{|l|}{ PEDro } \\
\hline $\begin{array}{l}\text { Therapy respiratory and } \\
\qquad \begin{array}{r}\text { Pneum } \\
\text { Lung } A \\
\text { Pneum } \\
\text { Lung } C \\
\text { Bullous }\end{array}\end{array}$ & $\begin{array}{l}\text { ele } \\
\text { ess } \\
\text { g disease }\end{array}$ \\
\hline
\end{tabular}


Foram incluídos nesta revisão estudos que utilizaram técnicas e recursos fisioterapêuticos respiratórios em crianças, adolescentes ou adultos diagnosticados com pneumatocele ou abcesso pulmonar. Estudos não disponíveis para acesso por meio do Programa de Comutação Bibliográfica (Comut), pelo website do jornal ou pelos bancos de dados Medline e Ebsco não fizeram parte do material analisado.

O método de triagem das publicações envolveu três examinadores, que analisaram os resultados da pesquisa de forma independente, na busca de estudos potencialmente elegíveis. Foram selecionados os trabalhos que respeitaram os critérios pré-estabelecidos, sendo discutidos os casos duvidosos. Inicialmente, os títulos foram armazenados em arquivos, separadamente, de acordo com a base de dados de origem. Verificaram-se possíveis repetições de títulos entre as bases de dados; e, na sequência, seguiu-se a análise.

\section{RESULTADOS}

Por intermédio das estratégias de busca, foi encontrado um total de 988 títulos sobre o assunto, sendo 398 títulos do PubMed (via Ovid), 428 da base de dados Lilacs e 162 da base de dados PEDro. Destes, 93 títulos foram compatíveis com o tema específico, e apenas 16 foram elencados para a análise dos resumos. Dos 16 resumos, 6 não se enquadraram aos critérios de inclusão ${ }^{20-25}$, restando 10 artigos para leitura na íntegra. Foram excluídos três trabalhos por não estarem disponíveis na íntegra via $\mathrm{Comut}^{26-28}$, e um estudo por não se enquadrar nos critérios inclusão ${ }^{29}$, pois não se referia apenas a pneumonia complicada, mas também incluía crianças como asma e refluxo gastroesofágico. A amostra final foi então composta por seis estudos $^{15,30-34}$

A busca conduzida na presente revisão identificou seis artigos ${ }^{16,30-34}$ que atenderam aos critérios de inclusão propostos, nos quais se verificou a utilização de técnicas de fisioterapia respiratória no tratamento dos indivíduos com pneumatocele.

Dos seis artigos incluídos, a maioria caracterizou-se como relato de casos $^{31-}$ ${ }^{34}$, e três ${ }^{32-34}$ foram publicados nos últimos 10 anos. Apenas o estudo de Paula et al..$^{32}$ foi realizado com crianças, e quatro estudos selecionados ${ }^{16,30,31,34}$ apresentavam amostras compostas por adultos; sendo que, em uma pesquisa ${ }^{32}$, não foi possível identificar a idade dos participantes. A frequência do tratamento fisioterapêutico não foi descrita em três estudos ${ }^{16,31,32}$; nos demais ${ }^{16,30,34}$, a terapia aconteceu três vezes na semana.

A técnica fisioterapêutica mais utilizada no tratamento do empiema e/ ou abcesso foi a drenagem postural ${ }^{16,30-34}$, sendo que três estudos ${ }^{31-34}$ avaliaram a associação dela com outras técnicas fisioterapêuticas, como a percussão, vibração manual, higiene de vias aéreas superiores e o instrumental de oscilometria oral de alta frequência. A antibioticoterapia esteve combinada em três pesquisas ${ }^{16,30-32}$. 
Figura 2. Fluxograma de seleção dos artigos

$\frac{5}{\text { Parazzi et al }}$

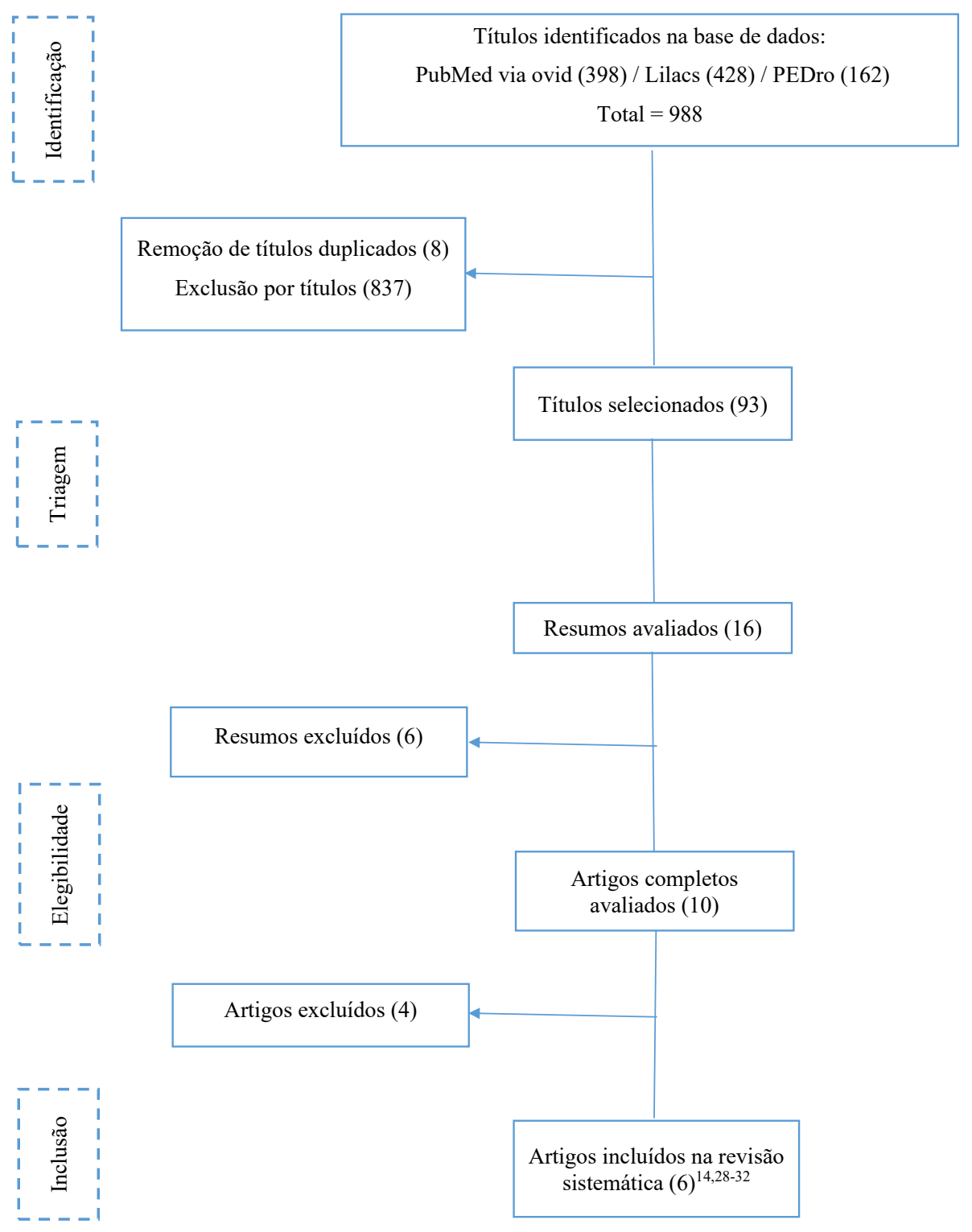


Para apresentação do conteúdo dos seis trabalhos selecionados, elaborou-se um quadro com informações referentes a amostra, idade, intervenção e resultados dos procedimentos fisioterapêuticos aplicados nos indivíduos com pneumatocele (quadro 1).

Quadro 1. Síntese dos trabalhos incluídos na presente revisão

\begin{tabular}{|c|c|c|c|c|c|c|}
\hline Estudo & Ano & Amostra & Idade & $\begin{array}{l}\text { Frequência } \\
\text { fisioterapia }\end{array}$ & Terapia & Resultados \\
\hline $\begin{array}{l}\text { Neild, et } \\
\text { al. }{ }^{30}\end{array}$ & 1985 & $\begin{array}{l}48 \text { adultos } \\
17 \text { abcesso (12 } \\
\text { o, } \quad 5 \text { + ); } \quad 31 \\
\text { empiema } \quad(22 \\
\text { o, } 9 \text { 9 }) .\end{array}$ & $\begin{array}{l}\text { Abcesso - } \\
\text { média } 47,6 \text { anos } \\
\text { Empiema - } \\
\text { média } 59,4 \text { anos }\end{array}$ & $3 x$ ao dia. & DP e antibióticos. & $\begin{array}{l}\text { - Abcesso: } 11 \text { pacientes } \\
\text { evidência radiológica mostrou } \\
\text { desparecimento da cavidade } \\
\text { e RX tórax mostrou resolução } \\
\text { completa em menos de } 4 \\
\text { meses. } \\
\text { - Empiema: } 1 \text { paciente com } \\
\text { melhora no RX pós } \\
\text { fisioterapia e antibióticos. }\end{array}$ \\
\hline $\begin{array}{l}\text { Rãsãnen et } \\
\text { al. }{ }^{31}\end{array}$ & 1988 & 1 Adulto $\widehat{\jmath}$ & 46 anos & $\begin{array}{ll}\text { Não foi } \\
\text { descrita. }\end{array}$ & $\begin{array}{l}\text { Percussões, DP, vibração } \\
\text { manual e tosse. }\end{array}$ & $\begin{array}{l}\text { - Diminuição de atelectasia } \\
\text { basilar direita. }\end{array}$ \\
\hline $\begin{array}{l}\text { Carvalho et } \\
\text { al. }{ }^{32}\end{array}$ & 1991 & $\begin{array}{ll}\text { Não } & \text { foi } \\
\text { descrito. } & \end{array}$ & Não foi descrito. & $\begin{array}{l}\text { Não foi } \\
\text { descrita. }\end{array}$ & $\begin{array}{l}\text { Inaloterapia sem } \\
\text { medicação antibiótica ou } \\
\text { fluidificante, DP seletiva, } \\
\text { cinesioterapia respiratória } \\
\text { (padrões respiratórios), } \\
\text { aspiração, espirometria } \\
\text { de incentivo. }\end{array}$ & $\begin{array}{l}\text { - Maior eficácia com tosse e } \\
\text { exercícios respiratórios nos } \\
\text { pacientes colaborativos. }\end{array}$ \\
\hline $\begin{array}{l}\text { Moreira et } \\
\mathrm{al}^{16}\end{array}$ & 2006 & $\begin{array}{l}209+9 \\
43 \hat{\gamma}\end{array}$ & $\begin{array}{l}\text { Média de } 41,4 \\
\text { anos }\end{array}$ & $\begin{array}{ll}\text { Não foi } \\
\text { descrita. }\end{array}$ & $\begin{array}{l}98,4 \% \text { dos pacientes } \\
\text { foram tratados com } \\
\text { antibiótico e DP. }\end{array}$ & $\begin{array}{l}\text { - Estabilidade e lou melhora } \\
\text { no quadro pulmonar. }\end{array}$ \\
\hline $\begin{array}{l}\text { Davidson } \\
\text { et } \mathrm{al}^{33}\end{array}$ & 2010 & $\begin{array}{l}1 \text { Adulto }{ }^{\hat{\sigma}} \\
1 \text { Adulto }{ }_{+}\end{array}$ & $\begin{array}{l}30 \text { anos } \\
28 \text { anos }\end{array}$ & $\begin{array}{l}1^{\circ} 3 \times / \text { dia } \\
\text { (14 dias). } \\
2^{\circ} 7 \text { dias. }\end{array}$ & $\begin{array}{l}\text { DP (trendelenburg } 15^{\circ} \text { a } \\
45^{\circ} \text { gradativamente) e } \\
\text { ERD nos dois casos. }\end{array}$ & $\begin{array}{l}\text { - Diminuição de atelectasias } \\
\text { lobo inferior direito. }\end{array}$ \\
\hline $\begin{array}{ll}\text { Paula et } \\
\text { al. }^{34}\end{array}$ & 2010 & 1 Criança ${ }_{\text {q }}$ & 8 anos & $\begin{array}{l}2-3 x / \\
\text { semanais. } \\
\text { Total - } 88 \\
\text { sessões. }\end{array}$ & $\begin{array}{l}\text { Higiene nasal, DRR, VM e } \\
\text { manual, OOAF, TEF, } \\
\text { CAR, AFE, tapotagem e } \\
\text { DP. }\end{array}$ & $\begin{array}{l}\text { - Estabilidade do quadro } \\
\text { pulmonar. } \\
\text { - Diminuição da incidência de } \\
\text { infecções bacterianas e } \\
\text { internações. } \\
\text { - Melhora da ausculta, da } \\
\mathrm{SpO}_{2} \text { e do padrão respiratório. }\end{array}$ \\
\hline
\end{tabular}

Legenda: đ- masculino; + - feminino; DRR - desobstrução rinofaríngea retrógada; VM - vibração mecânica; OOAF - oscilação oral de alta frequência; TEF - técnica de expiração forçada; CAR - ciclo ativo da respiração; AFE - aumento do fluxo expiratório; DP drenagem postural; ERD - exercícios respiratórios diafragmáticos; $\mathrm{SpO} 2$ - saturação periférica de oxigênio. 


\section{DISCUSSÃO}

A presente revisão identificou seis artigos16,30-34 que utilizaram técnicas de fisioterapia respiratória no tratamento dos indivíduos com pneumatocele. Dos seis artigos incluídos, a maioria caracterizou-se como relato de casos31-34, e somente um estudo, Paula et al.32, foi realizado com crianças, e quatro estudos 16,30,31,34 apresentavam amostras compostas por adultos. A técnica fisioterapêutica mais utilizada no tratamento do empiema e/ou abcesso foi a drenagem postural ${ }^{16,30-34}$, sendo que três estudos $^{31-34}$ avaliaram a associação dela com outras técnicas fisioterapêuticas.

No entanto, há uma escassez de estudos na área de fisioterapia respiratória no manejo de indivíduos com pneumatocele, revelando carência de pesquisas envolvendo métodos controlados para avaliação dos efeitos de diferentes intervenções fisioterapêuticas, e amostras extremamente limitadas. Além disso, observa-se ausência de padronização quanto à descrição das técnicas utilizadas, bem como de um consenso quanto à indicação delas.

Conforme a literatura, as pneumatoceles têm resolução completa dentro de semanas, e dificilmente deixam sequelas. Porém, as que persistem precisam ser tratadas com medicações específicas quando descendem de processos infecciosos, e uma pequena parte delas é submetida a técnicas invasivas ${ }^{5,16}$. O tratamento conservador tem se mostrado eficiente no seu acompanhamento, tendo a fisioterapia respiratória um papel importante na diminuição de sintomas e melhora da qualidade de vida dos indivíduos acometidos por essa complicação $0^{16,19}$.

A diversidade de nomenclatura descrita para designar a mesma doença também dificulta a busca de estudos sobre o tema. Identificou-se uma lista de palavras que incorporam essa enfermidade; além do termo pneumatocele, foram encontrados similares como: cisto pulmonar, doença pulmonar bolhosa, empiema pulmonar, pneumonias complicadas e abscesso pulmonar. Todos esses são apontados para caracterizar a presença de bolhas cheias de ar, com ou sem processo infeccioso no interior de sua cavidade, por isso foram a base da estratégia de busca desenvolvida no corrente artigo.

A técnica de fisioterapia respiratória apresentada com maior frequência no material selecionado foi a drenagem postural. Atribuiu-se a ela o objetivo de promover a desobstrução das vias aéreas com a vantagem de não oferecer riscos de rompimento dos cistos e eventual agravamento dos casos.

O estudo realizado por Neild e colaboradores $^{30}$ acompanhou 48 pacientes que apresentaram abcesso pulmonar/ empiema por um período de oito anos. $\mathrm{O}$ tratamento foi baseado em antibióticos e fisioterapia respiratória com drenagem postural três vezes por dia, e os autores consideraram o tratamento satisfatório para resolução e drenagem do abcesso.

$\mathrm{O}$ relato de caso de Räsänen et al. ${ }^{31}$ apresentou técnicas convencionais de fisioterapia respiratória, como drenagem postural, além de percussões e tosse. Os autores consideraram os efeitos dessas condutas como benéficas, mostrando-se eficaz no tratamento da hipersecreção em vias aéreas, associada ao quadro da pneumatocele, e não repercutindo na disseminação da infecção. Ainda nessa linha, Davidson et al. ${ }^{33}$ também descreveram os casos de dois pacientes submetidos a técnicas convencionais. A terapia constituiu-se de colocá-los em trendelenburg gradativamente alcançando os $45^{\circ}$ de inclinação; além disso, nos intervalos dos atendimentos, também se conduziram exercícios respiratórios diafragmáticos, identificando-se sinais positivos na desobstrução das vias aéreas evidenciando melhora da pneumatocele.

Moreira et al. ${ }^{16}$ relataram 252 casos de pneumatocele infecciosa (209 em homens) causada por aspiração de microrganismo, sendo que $82,2 \%$ desses casos apresentaram histórico de doença dentária. Os indivíduos foram monitorados por exames de imagens, análises bacteriológicas e hemogramas, sendo que todos os pacientes foram submetidos a tratamento com antibióticos e a aplicação da drenagem postural 
como técnica de fisioterapia respiratória. A maioria dos casos respondeu bem ao tratamento clínico conservador, e apenas 0,6 $\%$ dos pacientes tiveram complicações da pneumatocele.

A análise dos efeitos da aplicação de técnicas fisioterapêuticas modernas apresentada no relato de caso de Paula et al. ${ }^{33}$ descreve os resultados dessa terapêutica em uma paciente pediátrica com diagnóstico de síndrome da imunodeficiência adquirida e quadro de comprometimento motor e pulmonar. A criança teve diagnóstico de pneumonia intersticial crônica inespecífica, leve e focal, e história de 11 episódios de exacerbação. Os exames de imagem, radiografias e exames de tomografia computadorizada evidenciaram presença de pneumatocele gigante. A conduta fisioterapêutica teve como intuito manter a integridade pulmonar da paciente e aumentar a depuração mucociliar de forma segura. Para isso, aplicou-se a técnica de aumento do fluxo expiratório, ciclo ativo da respiração e técnica de expiração forçada. A opção por essa conduta foi justificada pelas autoras pelo fato de estas serem técnicas de desobstrução de vias aéreas menos agressivas e potencialmente capazes de prevenir exacerbações e piora do quadro respiratório, o qual pode acontecer mediante a ruptura da bolha e/ou instalação de processo infeccioso na cavidade.

Vale ressaltar que o tratamento conservador direciona para a resolução espontânea da pneumatocele dentro de poucas semanas, sem necessidade de intervenção cirúrgica. Os efeitos positivos da fisioterapia respiratória como coadjuvante no tratamento dessa afecção são alcançados, segundo Davidson et al. ${ }^{33}$, quando técnicas de higiene brônquica são bem empregadas. Entre estas, a drenagem postural é a única técnica descrita nos quatros estudos selecionados por ser considerada segura quanto ao risco de ruptura na parede da pneumatocele, e consequentes complicações como pneumotórax.

Como limitação do nosso estudo, consideramos a necessidade de mais estudos devido a escassez na área de fisioterapia respiratória no manejo de indivíduos com pneumatocele, pouca abordagem de diferentes técnicas e suas associações e amostras limitadas.

\section{CONCLUSÕES}

$\frac{8}{\text { Parazzi et al }}$

A presente revisão de literatura identificou poucos estudos referentes ao tratamento fisioterapêutico respiratório no manejo de indivíduos com pneumatocele. Os trabalhos apresentam efeitos benéficos de algumas técnicas de fisioterapia respiratória; no entanto, novas pesquisas devem ser conduzidas nessa linha, preferencialmente controladas, com amostras satisfatórias e padronização das técnicas.

Considerando o número limitado de artigos identificados, sendo a maioria relatos de casos, fica evidente a necessidade de estudos com delineamentos metodológicos mais detalhados, pois eles viabilizarão a indicação e a contraindicação de procedimentos fisioterapêuticos, auxiliando a tomada de decisão clínica perante essa terapêutica no tratamento da pneumatocele.

\section{REFERÊNCIAS}

1. Fletcher MA, Schimitt HJ, Syrochkina M, Sylvester G. Pneumococcal empyema and complicated pneumonias: globaltrends in incidence, prevalence and serotype epidemiology. Eur J Clin Microbiol Infect Dis. 2014;33(6):879-910.

2. Ehsan Z, Nata JD, Kercsmar CM. An infant with a hyperlucent chest mass: an unexpected diagnosis. Pediatr Pulmonol. 2015;50(12):E52-54.

3. Lai SH, Wong KS, Liao SL. Value of lung ultrasonography in the diagnosis and outcome prediction of pediatric community-acquired pneumonia with necrotizing change. PLoS One. 2015;10(6):e0130082.

4. Jimenez M, Singh R. Pneumonia amongst pneumonias: a persistente pneumatocele. Chest. 2012;142:4.

5. Kunyoshi V, Cataneo DC, Cataneo AJ. Complicated pneumonias with empyema 
and/or pneumatocele in children. Pediatr Surg Int. 2006;22(2):186-90.

6. Hoorebeke EV, Jorens PG, Wojciechowki M, Salgado R, Desager K, Schil $\mathrm{PV}$, et al. An unusual case of traumatic pneumatocele in a nine-year-old girl: a bronchial tear with clear bronchial laceration. Pediatr Pulmonol. 2009;44(8):826-8.

7. Fujii AM, Moulton S. Percutaneous catheter evacuation of a pneumatocele in an extremely premature infant with respiratory failure. J Perinatol. 2003;23(6):516-8.

8. Kumar J, Mukhopadhyay K, Bhatia A. Successful percutaneous drainage of pneumatoceles in an extremely low-birthweight infant. BMJ Case Rep. 2018:bcr2017222630.

9. Dibardino DJ. Index of suspicion for pneumatocele formation and awareness of management is not just for the trauma surgeon. J Am Coll Surg. 2005:318-319.

10. Mallavarapu RK, Katner HP. Pneumatocele complicating acute hydrocarbon pneumonitis. Clin Toxicol (Phila). 2008;46(9):911.

11. Yang TC, Huang CH, Yu JW, Hsieh FC, Huang YF. Traumatic pneumatocele. Pediatr Neonatol. 2010;51(2):135-138.

12. Jackson CC, Bettolli M, De Carli C, Rubin S, Sweeney B. Thoracoscopic treatment of a neonatal traumatic pneumatocele. J Laparoendosc Adv Surg Tech A. 2008;18(1):170-3.

13. Holland ET, Saulsbury FT. Chronic Pneumocystis carinii pneumonia associated with extensive pneumatocele formation in a child with human immunodeficiency virus infection. Pediatr Pulmonol. 2003;35(2):144-6.

14. Armstrong LB, Mooney DP. Pneumatoceles in pediatric blunt trauma: Common and benign. J Pediatr Surg. 2017: (17)30465-7.

15. Diretrizes brasileiras em pneumonia adquirida na comunidade em pediatria. J Bras Pneumol. 2007;33(1):S31-S50.

16. Moreira JS, Camargo JJP, Felicetti JC, Goldenfun PR, Moreira ALS, Porto, NS. Abscesso pulmonar de aspiração: análise de 252 casos consecutivos estudados de 1968 a 2004. J Bras Pneumol. 2006;32(2):136-43.

17. Kaira K, Ishizuka T, Yanagitani N, Sunaga N, Hisada T, Mori M. Pulmonary traumatic pneumatocele and hematoma. Jpn J Radiol. 2009;27(2):100-2.

18. Maimon NM, Day A. Traumatic pneumatocoele. Respiratology. 2007;12(4):617-8.

19. Imamoglu M, Cay A, Kosucu P, Ozdemir O, Cobanoglu U, Orhan F et al. Pneumatoceles in postpneumonic empyema: an algorithmic approach. J Pediatr Surg. 2005;40(7):1111-7.

20. Islam S, Calkins CM, Goldin AB, Chen $\mathrm{C}$, Downard CD et al. The diagnosis and management of empyema in children: a comprehensive review from the APSA Outcomes and Clinical Trials Committee. J Pediatr Surg. 2012;47(11):2101-10.

21. Tumwine JK. Lung abscess in children in Harare, Zimbabwe. East Afr Med J. 1992;69(10):547-9.

22. Egorova AI, Novikov VN, Krasavina NA. Diagnosis and treatment of complicated acute pneumonia in children. Pediatriia.1990;(5):94.

23. Bulynin VI, Kosheley PI, Barsukov VA. Treatment of acute lung abscess using hyperbaric oxygenation. Grud Serdechnososudistaia Khir. 1990;(5):37-41.

24. Kosloske AM, Ball WS, Butler C, Musemeche CA. Drainage of pediatric lung abscess by cough, catheter, or complete resection. J Pediatr Surg. 1986;21(7):596-600.

25. Narchi H. Gammoh S. Multiple nodular pneumonitis in a three-week-old female infant. Pediatr Infect Dis J. 1999;18(5):471,485-6.

26. Chernobrovyi NP. Use of physiotherapy and therapeutic gymnastics in the conservative treatment of pulmonary suppuration. Vopr Kurortol Fizioter Lech Fiz Kult. 1971;36(6):541-4.

27. Barkov VA, Komiachilova AS, Smirnova GI, Namestnikov VV, Rogozina TV. Treatment of abscessing pneumonia 
by using combination physiotherapy.

Vopr Kurortol Fizioter Lech Fiz Kult.

Parazzi et al

1987;(2)13-6.

28. Barkov VA. Suppurative-destructive complications of acute pneumonia and the principles of their treatment. Ter Arkh. 1986;58(4):123-8.

29. Brunetto AF, Paulin E. Importância da fisioterapia pulmonar no tratamento de pneumonias em crianças. Fisioter Mov. 2002;15(1):39-45.

30. Neild JE, Eykyn SJ, Phillips I. Lung Abscess and Empyema. QJM. 1985;57(224):875-882.

31. Rãsãnen J, Bools JC, Downs JB. Endobronchial drainage of undiagnosed lung abscess during chest physical therapy a case report. Phys Ther. 1988;68(3):371-373.

32. Carvalho LV, Vasconcelos RAS, Vargas RLAC, Spósito MMM, Jardim JRB. Lung abcess: pshysical therapy. Acta Paul Enferm. 1991;4(1):45-47.

33. Davidson J, Teixeira PT, Peixoto PFS, Crivellaro CM, Marques MA. Segurança e efetividade da fisioterapia respiratória em abscesso pulmonar: estudo de casos. Fisioter Pesqui. 2010;17(4):362-5.

34. Paula SRM, Gurgel MP, Macchiaverni L, Pereira FM, Ribeiro MAGO, Santos CIS. Pneumonia complicada por pneumatocele gigante em criança com síndrome da imunodeficência adquirida: importância da fisioterapia respiratória. Rev Paul Pediatr. 2010;28(2):244-248.

Recebido: 22/07/2020 Aprovado: 16/12/2020 